Fabrication and electrical integration of robust carbon nanotube micropillars by self-directed elastocapillary densification

This article has been downloaded from IOPscience. Please scroll down to see the full text article.

2011 J. Micromech. Microeng. 21045033

(http://iopscience.iop.org/0960-1317/21/4/045033)

View the table of contents for this issue, or go to the journal homepage for more

Download details:

IP Address: 141.211.173.82

The article was downloaded on 06/04/2012 at 16:52

Please note that terms and conditions apply. 


\title{
Fabrication and electrical integration of robust carbon nanotube micropillars by self-directed elastocapillary densification
}

\author{
Michaël F L De Volder ${ }^{1,2,3}$, Sei Jin Park ${ }^{1}$, Sameh H Tawfick ${ }^{1}$, \\ Daniel O Vidaud ${ }^{1}$ and A John Hart ${ }^{1}$ \\ ${ }^{1}$ Department of Mechanical Engineering, University of Michigan, 2350 Hayward Street, Ann Arbor, \\ MI 48109, USA \\ ${ }^{2}$ IMEC, Kapeldreef 75, 3001 Heverlee, Belgium \\ ${ }^{3}$ Department of Mechanical Engineering, Katholieke Universiteit Leuven, 3001 Leuven, Belgium \\ E-mail: ajohnh@umich.edu and michael.devolder@imec.be
}

Received 8 September 2010, in final form 22 February 2011

Published 24 March 2011

Online at stacks.iop.org/JMM/21/045033

\begin{abstract}
Vertically aligned carbon nanotube (CNT) 'forest' microstructures fabricated by chemical vapor deposition (CVD) using patterned catalyst films typically have a low CNT density per unit area. As a result, CNT forests have poor bulk properties and are too fragile for integration with microfabrication processing. We introduce a new self-directed capillary densification method where a liquid is controllably condensed onto and evaporated from the CNT forests. Compared to prior approaches, where the substrate with CNTs is immersed in a liquid, our condensation approach gives significantly more uniform structures and enables precise control of the CNT packing density. We present a set of design rules and parametric studies of CNT micropillar densification by self-directed capillary action, and show that self-directed capillary densification enhances Young's modulus and electrical conductivity of CNT micropillars by more than three orders of magnitude. Owing to the outstanding properties of CNTs, this scalable process will be useful for the integration of CNTs as a functional material in microfabricated devices for mechanical, electrical, thermal and biomedical applications.
\end{abstract}

(Some figures in this article are in colour only in the electronic version)

\section{Introduction}

While the outstanding properties of carbon nanotubes (CNTs) $[1,2]$ have generally been verified by characterization of individual CNTs [3, 4], the collective electrical [5], mechanical [6] and thermal [7] properties of CNT assemblies have largely not satisfied predictions based on scaling laws. This shortcoming is mainly due to challenges in fabricating CNT assemblies, namely controlling the diameter, packing density and straightness of CNTs, and establishing robust electrical and mechanical contact with the CNTs. Further, the integration of CNT assemblies as a functional material for uses such as microelectronic interconnects and thermal interfaces $[5,7]$ requires parallel placement of large numbers of assemblies under conditions compatible with microfabrication.
The most common approach to fabricating CNT assemblies for use in microsystems is the growth of vertically aligned CNT 'forests' from a catalyst layer by chemical vapor deposition (CVD) [8, 9]. This approach enables vertical self-organization of CNTs on a silicon substrate, and this mechanically favorable configuration facilitates the growth of films comprising parallel CNTs whose length can reach up to several millimeters. Further, patterning of the catalyst layer by photolithography enables the fabrication of CNT forest microstructures having virtually any cross-sectional shape. The lateral dimensions of CNT forest microstructures are limited by the lithography resolution, and their aspect ratio (height divided by diameter) is limited by the growth process and catalyst lifetime [9]. Nevertheless, the dimensional limits of CNT forests grown by CVD are comparable to vertical microstructures made by top-down methods such as DRIE 


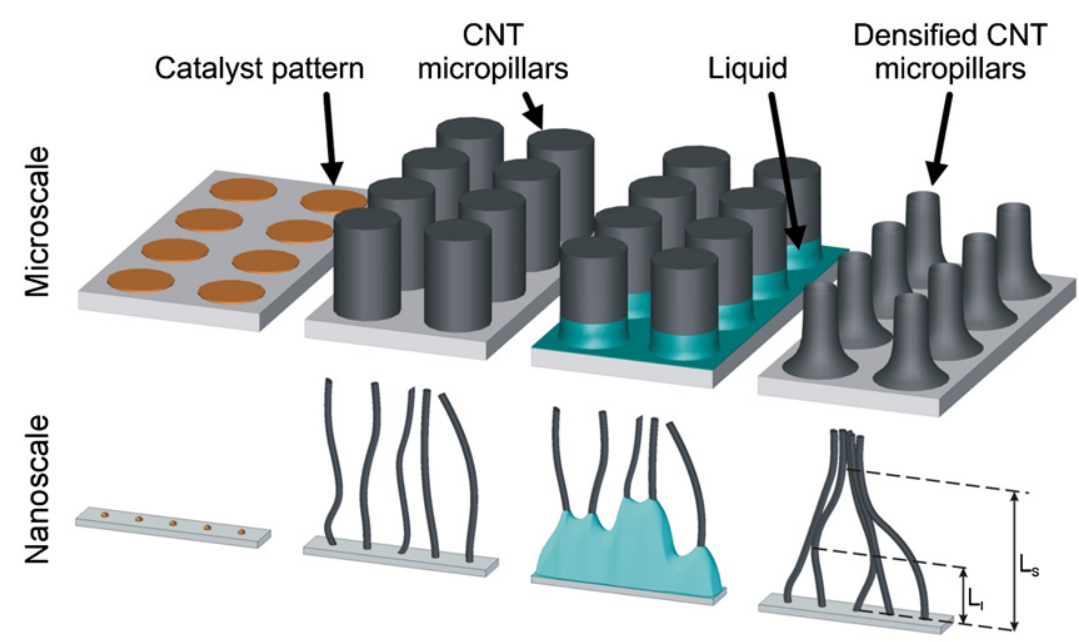

Figure 1. Fabrication of robust CNT micropillars by patterned growth followed by self-directed capillary densification. The densification is initiated by condensation of a solvent onto the substrate.

(deep reactive ion etching) [10]. Even at $10 \%$ of the ideal stiffness and strength of individual CNTs, the mechanical properties of CNT microstructures would rival those of silicon, and CNTs have high electrical and thermal conductivity. Microstructures made from materials with such attractive properties would enable major advances in microsystems technology.

Unfortunately, although CNT forest growth is well established, the bulk density of CNT forests is typically only $1-5 \%$, and therefore the bulk properties of the CNT forests are far below those of individual CNTs. Therefore, to increase the robustness of CNT forests, post-growth processing has been employed. The first approach is to densify CNT forests after growth, and there are two well-known densification methods: use of mechanical forces by compressing, shearing and/or rolling CNT forests during or after growth [11-14]; and use of capillary forces by submerging CNT forests in a liquid, and subsequently evaporating the liquid [15-19]. The second approach is to infiltrate CNT forests with a polymer or ceramic matrix material thus creating a nanocomposite microstructure, via a liquid-phase method such as capillary infiltration epoxy [20], or a vapor-phase approach such as CVD of a polymer or ceramic [21-23]. While the resulting composites have shown much greater mechanical properties than as-grown forests, direct infiltration of polymers is not suitable for small CNT microstructures due to forces exerted by the flow of the uncured polymer, and coating the CNTs may not be desirable because it renders the CNT surfaces inaccessible for electrical contacting or further processing.

We introduce a new process for fabricating robust CNT microstructures for use in microsystems, wherein CNT forest microstructures are densified by self-directed capillary action. In the new self-directed capillary densification process, the densifying liquid is evaporated from a reservoir and condensed onto the CNT forests, as shown in figure 1(a). This condensation method enables the application of minute amounts of densifying liquid to the substrates, which infiltrates each structure independently and results in the individual densification of each structure without the formation of capillary bridges. Within each structure, the CNTs locally aggregate by the elastocapillary mechanism [24], and coupling among the aggregating CNTs within each structure causes the entire microstructure to densify. Compared to the immersion method, we show that the condensation method preserves much more delicate and higher aspect ratio structures (see figure 2). In addition, our method allows use of a wide range of densifying liquids, including highly viscous liquids that result in a higher densification factor. In separate publications, we recently showed that the self-directed densification method also facilitates design and fabrication of unique 3D CNT microstructures [25, 26], and that densified CNT microstructures can be used as master molds for polymer replication $[25,27]$. Herein, we develop a set of design criteria for the fabrication of robust CNT micropillars by the self-directed capillary method, and present the basic mechanical and electrical properties of the densified micropillars.

\section{Methods}

CNT forest microstructures are grown on thermally-oxidized (1 000$)$ silicon wafers, using a supported catalyst layer of $10 \mathrm{~nm} \mathrm{Al}_{2} \mathrm{O}_{3}$ and $1 \mathrm{~nm} \mathrm{Fe}$ deposited by e-beam evaporation [9]. The catalyst layers are patterned by a lift-off process using photoresist (SPR 220) and ultrasonic agitation in acetone. Next, CNTs are grown in a horizontal tube furnace (22 mm inner diameter, $300 \mathrm{~mm}$ heated length) at atmospheric pressure, with flows of $100 / 400 / 100 \mathrm{sccm} \mathrm{C}_{2} \mathrm{H}_{4} / \mathrm{H}_{2} / \mathrm{He}$, at $775{ }^{\circ} \mathrm{C}$. The growth time is typically 0.5 to $15 \mathrm{~min}$ depending on the desired forest height. Optionally, the adhesion of the CNTs to the substrate is enhanced by rapidly cooling the substrates in the growth atmosphere immediately after the conclusion of the programmed growth time [13]. In this case, the furnace hood is raised with the carbon source still flowing. This recipe results in multi-wall CNTs (MWCNTs) with average diameter $10 \mathrm{~nm}$. In some experiments, the CNTs are plasma etched prior to densification, using $10 \mathrm{sccm} \mathrm{O}_{2}$ at a 
(a)

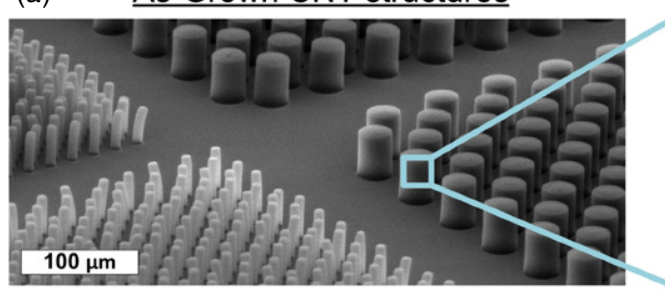

(c)

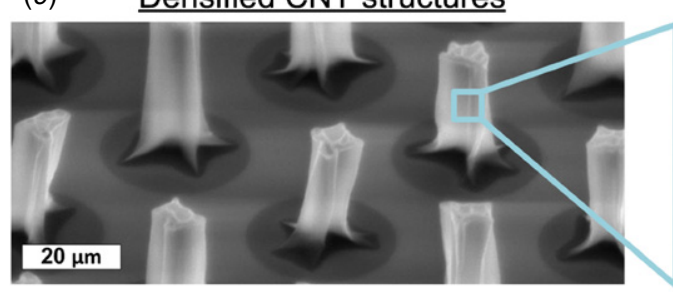

(e)

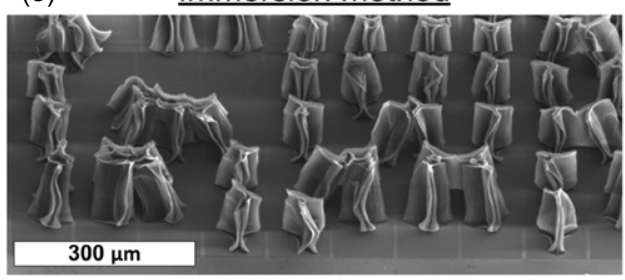

(b)

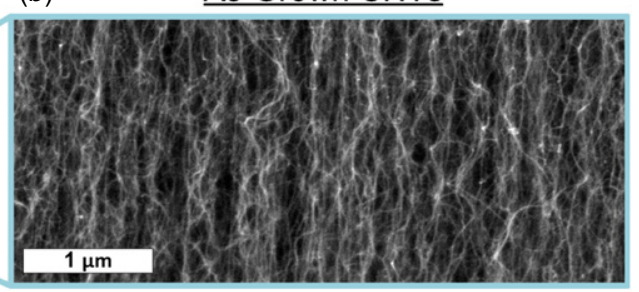

$(d)$

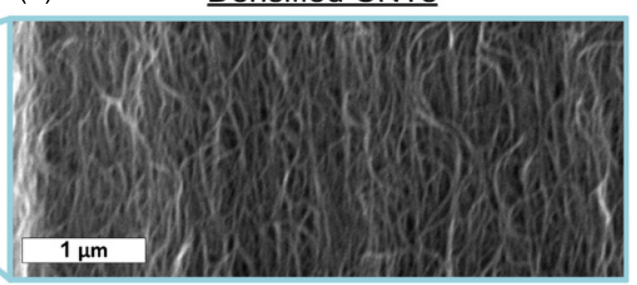

(f) $\quad$ Condensation method

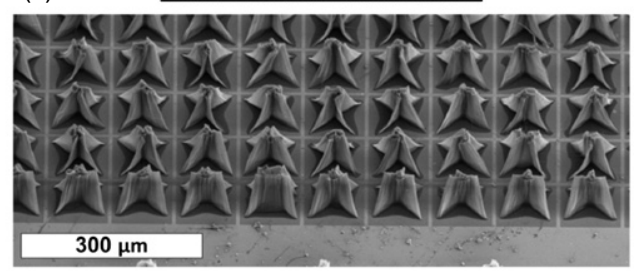

Figure 2. Example SEM images of cylindrical CNT microstructures before $(a, b)$ and after densification ( $c$, $d$ ) by the self-directed method; and comparison between results of the self-directed method $(e)$ and the immersion method $(f)$. Close-up images $(b, d)$ show the sidewalls of the microstructures, emphasizing the significant increase in CNT density and the maintenance of the aligned topology.

pressure of $75 \mathrm{mTorr}$ and $80 \mathrm{~W}$ power, for $30 \mathrm{~s}$. This removed the tangled 'crust' at the top of the forest (see later).

For the capillary densification process, the silicon substrate with CNTs is placed on an aluminum mesh (McMaster-Carr 9232T221) and inverted over a large beaker (1 L) containing a small amount of liquid (e.g., $20 \mathrm{~mL}$ acetone), as illustrated in figure 3 . The aluminum mesh is covered with a lab towel (e.g., a polypropylene/cellulose composite wiper, Fisher Scientific) to absorb droplets of liquid. The beaker is placed on a hotplate set to $175^{\circ} \mathrm{C}$ for acetone and $300{ }^{\circ} \mathrm{C}$ for glycerol. This setup produces an upward vapor flux and maintains a lower substrate temperature that drives condensation of the liquid onto the substrate. For basic process control, we leave the substrate in place until the liquid has fully evaporated, which typically takes less than $10 \mathrm{~min}$. The results shown in this paper were obtained using acetone and glycerol. We also performed experiments using water, but found that it did not enable uniform densification. This is likely due to the hydrophobicity of as-grown forests; water droplets condensed on the substrate but did not uniformly infiltrate the CNTs and instead deformed the structures.

The densification setup was placed under a snorkel vent or inside a fume hood, and the position of the sample was controlled carefully so that the samples were never 'pooled' by excessive vapor condensation. Enhanced control could be achieved using a chamber with a cold stage that operates at sub-atmospheric pressure, and therefore manipulates the conditions of the sample with respect to the vapor-liquid line on the solvent phase diagram.
In order to quantify the improvement in the density of the structures, we define a parameter called the densification factor (DF). The DF is defined as the ratio of the cross-sectional area of the microstructure before and after densification. Top-view SEM pictures are used to calculate the DF, by comparing the area of the catalyst to the top cross-sectional area of the structure after densification. The cross-sectional area before densification is assumed to be the same as the area of the catalyst. The measurements are made using INFINITY ANALYZE software.

\section{Results and discussion}

Figures 2(a) and (c) show typical CNT micropillars before and after densification using the self-directed capillary method. As shown in the close-up on the right-hand side of this figure, densification significantly increases the CNT packing density. Figures $2(e)$ and $(f)$ compare micropillars densified using the condensation and immersion methods. In the immersion method, the same solvent is used but the substrate is manually submerged in the solvent and then withdrawn and allowed to dry. As can be seen in these figures, immersion densification tends to damage closely spaced pillars, whereas the selfdirected method allows the structures to densify individually.

In what follows, we present a set of guidelines for the design and fabrication of robust densified microstructures. We will address a number of critical issues for the fabrication of robust CNT microstructures by capillary densification, such as the formation of internal voids and changes in the 


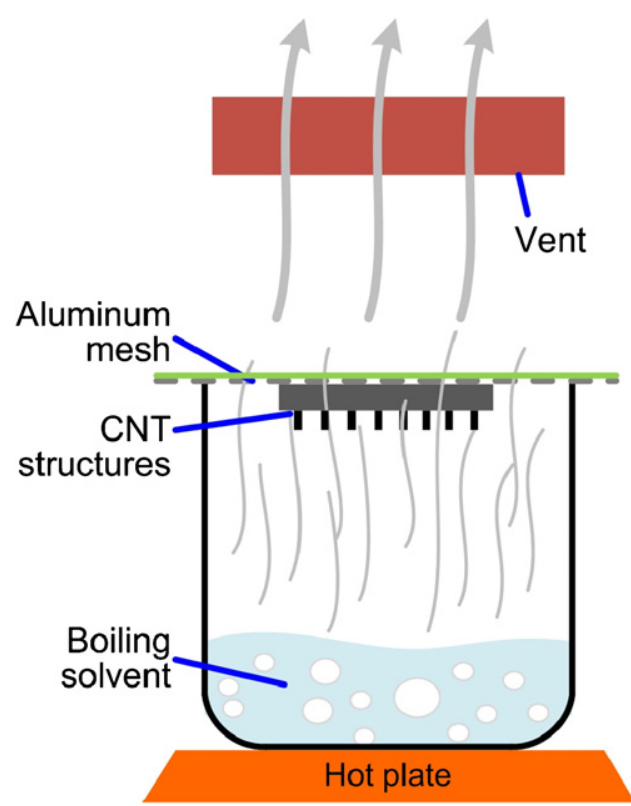

Figure 3. Setup for the densification of CNT forests by the self-directed method, where solvent vapor is condensed onto a substrate held above a large beaker.

cross-sectional shape (e.g. wrinkles in figure 2), and the dependence of the densification factor on the CNT pillar size and spacing. By variation of the CNT growth and densification process parameters, we demonstrate the tuning of the DF, and show that densified CNT pillars made by our process have mechanical stiffness and electrical conductivity more than 1000-fold higher than as-grown CNT forests.

\subsection{Control of void formation}

Elastocapillary aggregation occurs when an ensemble of slender beams is withdrawn from a liquid, and the resultant capillary forces between the liquid and the solid bring the beams closer together. Via immersion in liquids, this phenomenon has been studied for the aggregation of wet hair [24], nano- and microfibers [28, 29], polymer microstructures [30], and CNT forests [15, 31, 32]. If the capillary forces are stronger than the elastic restoring forces for the deformed beams, the beams will aggregate, and in the case of microand nanostructures, surface forces can make this aggregation stable after the liquid evaporates. When the structures are distributed over a large area, e.g., a non-patterned CNT forest, the contraction of the forest during the densification together with the adhesion of the CNTs to the substrate results in the formation of randomly distributed voids. The final structure then resembles an open-cell foam. When the structures are pre-patterned into smaller areas, e.g., an array of CNT microstructures, each microstructure may have internal voids, or each structure may form a single aggregate. Although the formation of voids can be useful for certain applications such as trapping of biological cells [17], void-free structures are prerequisite for achieving microstructures with a high CNT density.

This 'phase boundary' between the formation of voids and the formation of single aggregates depends on the dimensions of the CNT microstructures along with the diameter and density of the CNTs. We applied analytical models of elastocapillary aggregation to derive the geometrical requirements to form void-free CNT microstructures, and show that results from the self-directed capillary process achieve good agreement with this theory.

We consider a CNT forest to be an array of vertical beams that are rigidly connected to a substrate, having radius $R(5 \mathrm{~nm})$, spacing $d$ (averaging $100 \mathrm{~nm}$ ) and Young's modulus $E(1 \mathrm{TPa})$. Following Py et al [28], $L_{I}$ denotes the distance between the substrate and the point where two adjacent CNTs are pulled together (figure 1),

$$
L_{I}=\left(\frac{9}{2 \cdot(\pi-2)}\right)^{1 / 4} \sqrt{d \cdot \sqrt{\frac{E \cdot \pi \cdot R^{3}}{4 \cdot \gamma}}} .
$$

Here, $\gamma$ is the surface tension $\left(0.025 \mathrm{~N} \mathrm{~m}^{-1}\right.$ for acetone). For these model parameters, $L_{I}$ is approximately $400 \mathrm{~nm}$. If the length of the CNTs exceeds $L_{I}$, this pairing process will continue successively to form a hierarchical structure comprising $N$ CNTs which ultimately contact at a 'sticking distance' $L_{S}$ above the substrate. For the array of CNTs, the sticking distance is

$$
L_{S}=L_{I} \cdot\left(\frac{\beta^{2} \cdot(\pi-2)}{2 \sqrt{\pi} 3^{1 / 4}(2-\sqrt{2})}\right)^{1 / 4} \cdot N^{3 / 8} .
$$

Here, $\beta$ accounts for the lattice geometry and is set equal to 0.5 (see [28]). In practical terms, an arrangement of $N$ CNTs must be longer (taller) than $L_{S}$ in order to form a single aggregate, without internal voids. The cluster size $N$ can be determined using the cross-sectional area of the CNT microstructure and the areal density of the CNTs, which is known from the CNT diameter and the mass density of the forest [33].

Alternatively, Zhao et al $[15,31,32]$ derive the sticking distance as

$$
L_{S}^{3} \sim(N-1) \cdot R^{2} \cdot d^{2} \cdot\left(1-\sqrt{R^{2} / d^{2}}\right)^{2} .
$$

Finally, Journet et al reported a study of elastocapillary coalescence of non-patterned CNT forests that were wetted with a water droplet resulting in the formation of CNT 'huts' with diameter $\zeta$ and height $L$ [31],

$$
\zeta \sim \sqrt{\frac{\gamma \cdot L^{4}}{E \cdot R^{3}}} .
$$

These analytical methods can be applied to predict the formation of voids during capillary densification. In order to validate the applicability of the elastocapillary models for the design of CNT microstructures for densification, we performed a thorough dimensional study using the condensation process, using cylindrical CNT microstructures ('pillars') of varying diameter $(D)$ and height $(L)$. From these data, we plotted the 'phase transition' between the formation of a void-free densified structure and the formation of a structure with internal voids. As expected, the pillar height $\left(L_{S}\right)$ below which micro-voids are observed increases with the pillar diameter. Relatively short and wide pillars exhibit internal voids as the capillary forces cannot overcome the elastic strain necessary to bring all the CNTs together without the formation of microvoids. Relatively tall and narrow pillars form single aggregates 

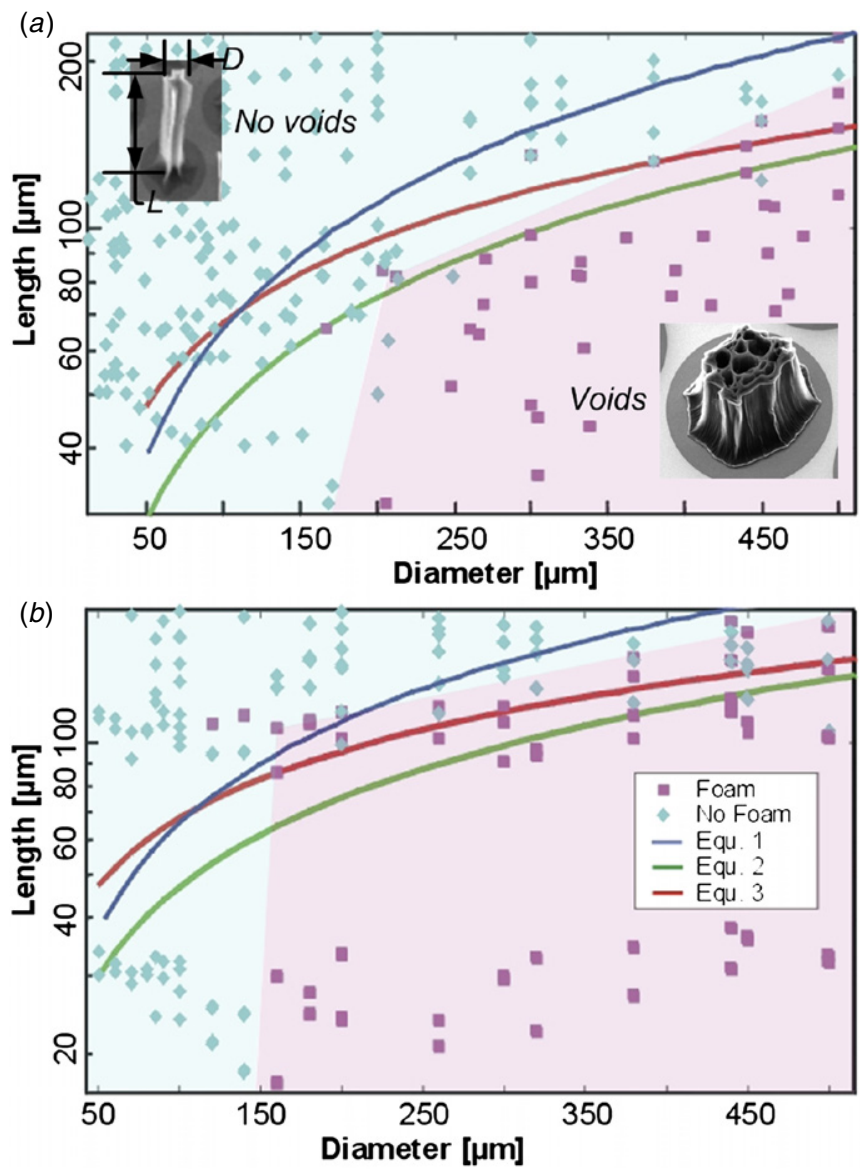

Figure 4. Effect of CNT microstructure dimensions on aggregate formation: (a) comparison of analytical models to measurements of cylindrical microstructures; and $(b)$ comparison of analytical models to structures that were plasma etched to remove the top crust prior to densification.

because less elastic deformation is required to bring the CNTs together, and because the CNTs have greater length to facilitate the hierarchical elastocapillary pairing process.

As illustrated in figure $4(a)$, the analytical models fit the experimental data well for CNT pillars with $D>200 \mu \mathrm{m}$. Several phenomena could explain the remaining discrepancies between our measurements and the above models. The models described above have been derived for ideally straight and uniformly spaced rods that contact only after action by capillary forces. However, as shown in figure 2, CNTs within a forest have significant waviness, and frequent contact. Second, in some cases the CNTs partially detach from the catalyst pattern as they are pulled together, which is not taken into account in the above models. Nevertheless, models that treat CNTs as elastic beams are a useful guide for selecting the CNT pillar dimensions for void-free densification.

Another important consideration is the tangled 'crust' layer on the top of CNT forests, which forms as the CNTs selforganize at the start of the growth process $[34,35]$. Because of its tangled morphology, the layer constrains lateral movement of the CNTs during densification. Therefore, we repeated the parametric study of aggregation, with structures where the crust layer was removed by plasma etching after growth. As
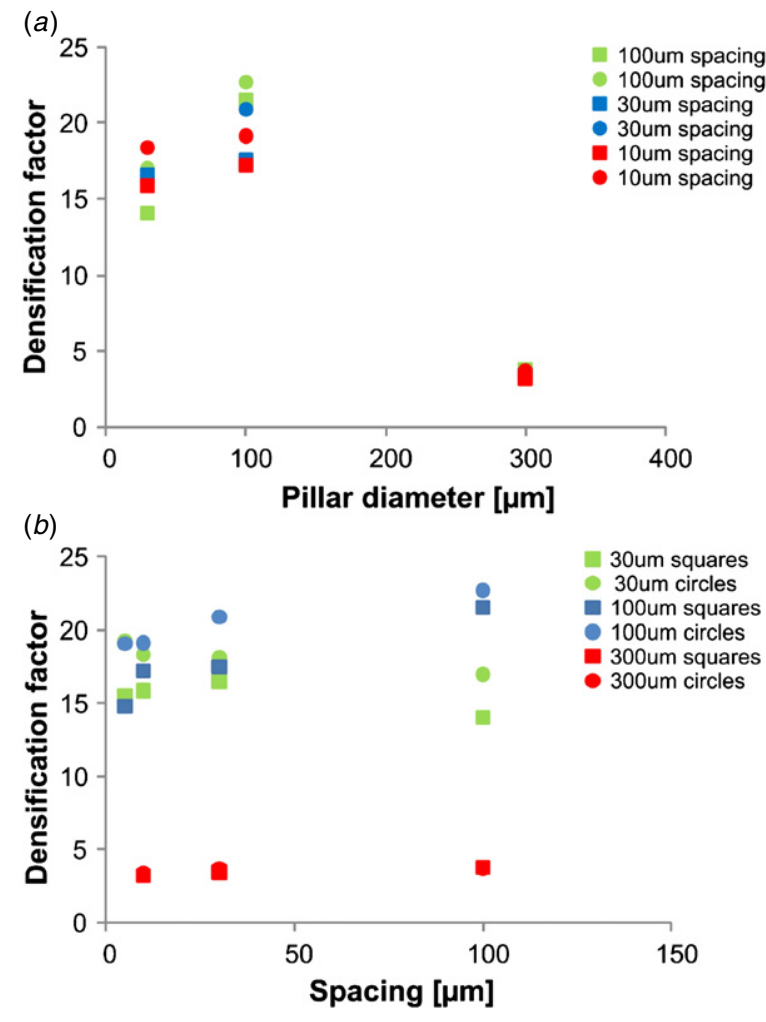

Figure 5. Densification factor of CNT pillars densified using glycerol as a function of $(a)$ pillar diameter, for several spacings; and $(b)$ pillar spacing, for several diameters. The shape of the data marker indicates the initial cross-sectional shape of the CNT pillar (circle or square). In the case of square pillars, the 'diameter' refers to the side length of the square. Typically, the standard deviation of the densification factor is $20 \%$ of the measured values.

shown in figure 4(b), the foam/no-foam boundary for the crustfree structures agrees with the elastocapillary models from a diameter of $150 \mu \mathrm{m}$ onward. This improved correlation is logical since the crust layer introduces a constraint that is not taken into account in the model.

\subsection{Control of the $D F$}

After defining geometric bounds for void-free densified microstructures, we now investigate how to control the packing density of CNTs by manipulating the balance between elastic and capillary forces during the densification process. While ideally the CNTs would aggregate in perfect closely packed clusters, the tortuosity of the CNTs within the forest and the presence of the crust prevent perfect packing. Further, the diameter and initial packing density of the CNTs determines the elastic resistance to densification, and the surface tension of the liquid and the dynamics of liquid delivery may affect the strength of the capillary forces. These variables can be manipulated by the CNT pattern design, the CNT growth parameters and the densification process parameters.

First, we study how the DF depends on the size and spacing of the CNT microstructures. Figure 5 shows the DF for glycerol densification as a function of the spacing for different pillar diameters and lengths, for both square and circular cross-sections. Within measurement error, the DF is 
invariant with the spacing. Small variations could arise from the effect of pillar spacing on the amount of available area for liquid condensation which affects the infiltration dynamics, and effects of the microstructure diameter on the CNT areal density. The latter topic is currently under investigation. The $\mathrm{DF}$ is $\approx 5$ for $300 \mu \mathrm{m}$ diameter pillars which form internal voids, yet is $15-20$ for 100 and $30 \mu \mathrm{m}$ diameter pillars which form a single aggregate. Note that the DF is calculated by dividing the initial cross-sectional area by the densified crosssectional area, without taking voids into account.

Further, differences in the strength of CNT-substrate adhesion can affect the DF. This is due to the fact that as the pillars get densified, some nanotubes around the edge may not be long enough to get tightly bound to the central cluster, hence preventing ideal shrinkage. If these nanotubes are detached from the substrate due to capillary forces, further shrinkage may occur. The adhesion of the CNTs to the substrate is therefore another important parameter for controlling densification.

Now for a fixed pillar size and spacing, we control the DF by varying the structural characteristics of the CNT micropillars. First, the diameter of the CNTs is controlled by the catalyst nanoparticle size. During the annealing step, the Fe film breaks into nanoparticles, which then seed the CNT growth. Thus, we found that annealing the catalyst in He without $\mathrm{H}_{2}$ yields larger particles and hence MWNTs with average diameter of $\approx 20 \mathrm{~nm}$ instead of $\approx 10 \mathrm{~nm}$. As illustrated in figure 6, CNT micropillars made out of $10 \mathrm{~nm}$ diameter CNTs yield a higher densification than $20 \mathrm{~nm}$ diameter CNTs. This is because the elastic stiffness of the CNTs is proportional to $D^{3}$, whereas the areal density is proportional to $1 / D^{2}$.

Figure 6 also shows the influence of the crust layer on the densification process. Removing the crust layer by brief $\mathrm{O}_{2}$ plasma etching (see section 2) after growth enables more slip among the CNTs during the densification process, and thereby results in higher densification and more uniform radial contraction. Figure 6 shows the influence of the plasma etching step on round pillars with a $100 \mu \mathrm{m}$ diameter using acetone. The plasma etch increases the DF by 2- to 4-fold, and results in a smoother contraction. The highest DF of approximately 30 is obtained with small diameter CNTs that were plasma etched. Taken together, the influence of the pillar diameter, spacing, CNT diameter and crust layer shows that the DF can be controlled from 4 to 30 . This is a packing fraction of approximately $6 \%$ to $48 \%$, relative to an ideal packing fraction of $100 \%$ defined for hexagonally arranged cylinders.

\subsection{Control of cross-sectional shape}

In most cases, the densification of CNT micropillars causes the cross-sectional shape to change. For instance, as the structures shrink, the sidewalls fold, resulting in wrinkled pillars as shown in figures 2 and 6 . The wrinkling of the structures could be induced by a difference in the amount of densification between the CNTs on the periphery and the core of the forest, or by the dynamics of wetting during liquid infiltration and evaporation. Less wrinkling is observed on structures that have been plasma etched before densification, as also shown in figure 6 .

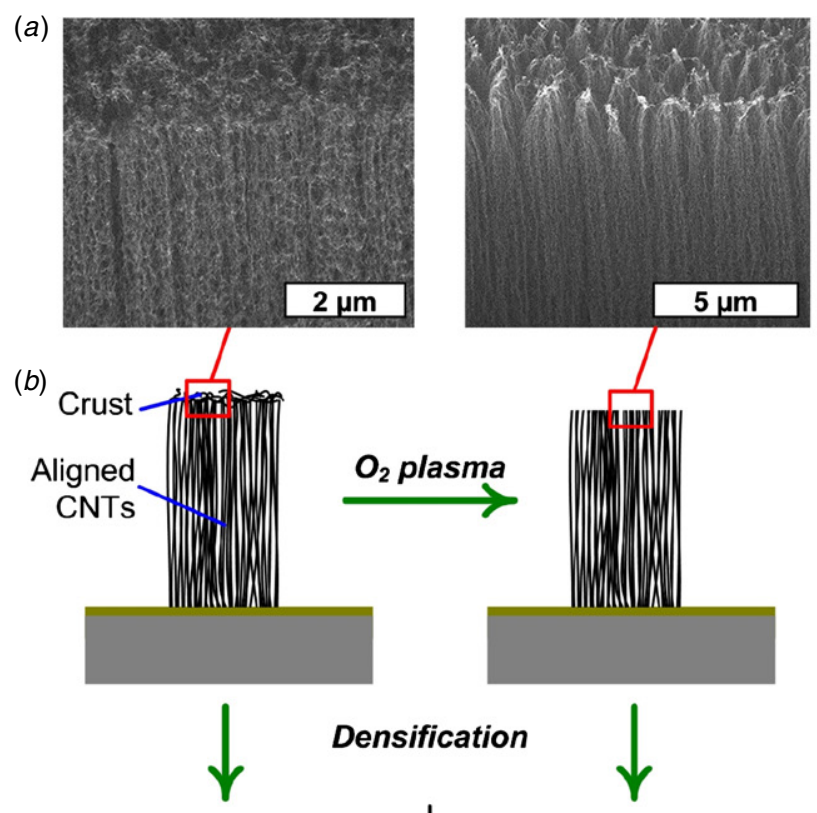

(c) CNT diameter $=10 \mathrm{~nm}$

$(d)$

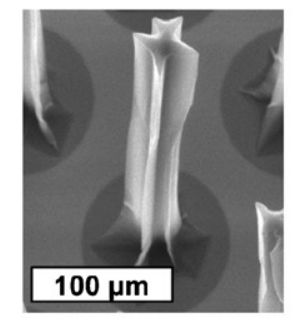

Densification $=7 \mathrm{X}$

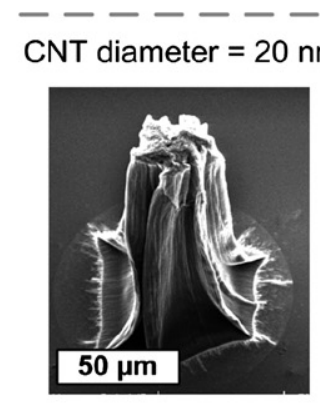

Densification $=6 \mathrm{X}$ CNT diameter $=10 \mathrm{~nm}$

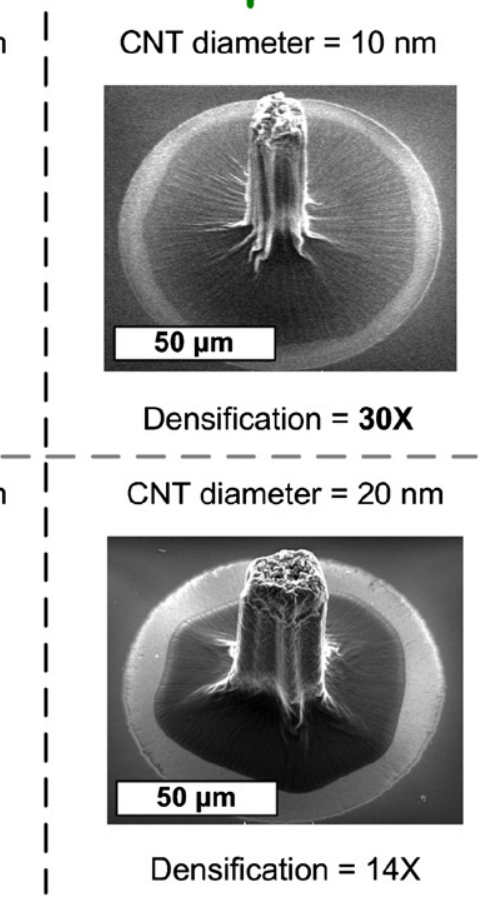

Figure 6. Influence of plasma etching and CNT diameter on the densification of cylindrical micropillars, using acetone: (a) SEM image of the crust layer on top of an as-grown micropillar; $(b)$ schematic of micropillars as-grown and after plasma etching which removes the crust; (c) SEM images of micropillars after densification, with CNT diameter $10 \mathrm{~nm}$; and (d) SEM images of micropillars after densification, with CNT diameter $20 \mathrm{~nm}$.

Further, we found that polygonal pillars form star-shaped cross-sections after densification as illustrated in figure 7 . The number of points and arrangement of the star is dictated by the number of corners in the catalyst pattern, although as more corners are added, some points are less clearly defined. Further, as the corners of the catalyst pattern get more rounded (figure $7(c)$ ), the corners in the cross-section of the densified pillars also become more rounded. Removing the crust by plasma etching (figures $7(b)$ and $(d)$ ) allows the sidewalls to 

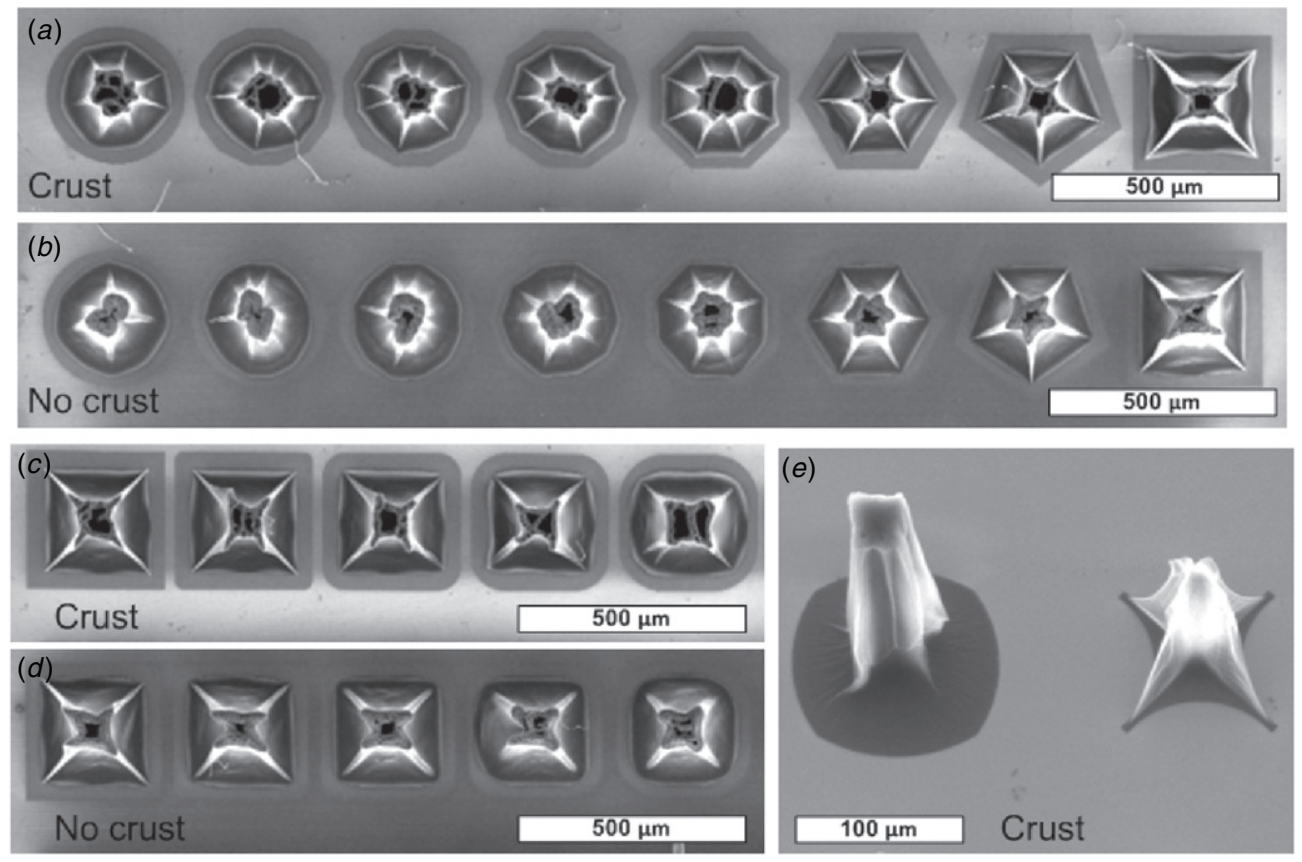

Figure 7. Effect of the catalyst pattern shape and the top crust on the pillar cross-section after densification: $(a)$ polygonal pillars; $(b)$ polygonal pillars where the crust was removed by plasma etching before densification; $(c)$ square pillars with rounded corners; $(d)$ square pillars with rounded corners, crust removed; and $(e)$ comparison of concave and convex catalyst patterns after densification (crust not removed).

contract more smoothly. Finally, the catalyst pattern can be designed to inhibit the formation of star-shaped structures. Figure 7(e) illustrates how concave shapes accentuate the formation of a star-shape, while convex catalyst patterns can cancel out this effect and result in pillars with a more rectangular cross-section after densification.

\subsection{Interactions among micropillars and the formation of hierarchical textures}

Understanding the limits to the spacing of microstructures is important for the fabrication of probe needle arrays and adhesive surfaces. Here we determine the geometric limits and discuss the formation of hierarchical textures from pillars that contact one another during growth and densification. To do so, 'phases' are defined that describe the overall pillar arrangement as illustrated in figure 8 . It is important to note from figure 8 that as-grown CNT pillars are not always perfectly straight. In the CNT fabrication process used in this paper, the CNT micropillars are limited to aspect ratios of 8 , above which the pillars start to bend due to growth variations. For an in-depth discussion of pre-densified arrangement of the pillars, we refer to an earlier publication [35]. In what follows we will only discuss how the densification process changes the arrangement or phase of the pillars. As we will see, the straightness of the as-grown CNT pillars affects their straightness after densification, and their proximity affects how the pillars interact during densification.

We use the same definitions of 'phases' as we introduced in the previous work [35].
- Phase 1: Micropillars grow vertically straight and have tip deflections of less than their own diameter. Adjacent pillars do not touch one another.

- Phase 2: Micropillars have tip deflections of at least one pillar diameter, yet the pillars are still self-supporting (i.e. tips of adjacent microstructures are not touching).

- Phase 3: Micropillars have tip deflections of at least one pillar diameter, and adjacent CNT pillars are touching one another.

- Phase 4: Micropillars are entwined and support one another, and remain suspended above the substrate after densification.

- Phase 5: Micropillars fail to grow vertically or fail to stay suspended after densification, forming a tangled arrangement of CNTs.

Clearly, pillars that are touching one another after growth (phase 3) will interact with each other during the densification process. Typically, menisci are formed that couple capillary forces among these structures, or between structures and the substrate. This process aggregates pillars or pulls them down, typically resulting in phase 4 or 5 (see figure 8). This effect is studied in more detail in figure 9, which plots the phases before and after densification for arrays of CNT pillars with a range of diameter and spacing values. The first column of figure 9 shows the phases before the densification process, and the second column shows the phases of the microstructures after densification using glycerol. The plots emphasize that pillars with higher aspect ratio have an increasing tendency to collapse during densification. Note that the closely spaced pillars retain a slightly higher phase because they support one another, which prevents them from falling down. 


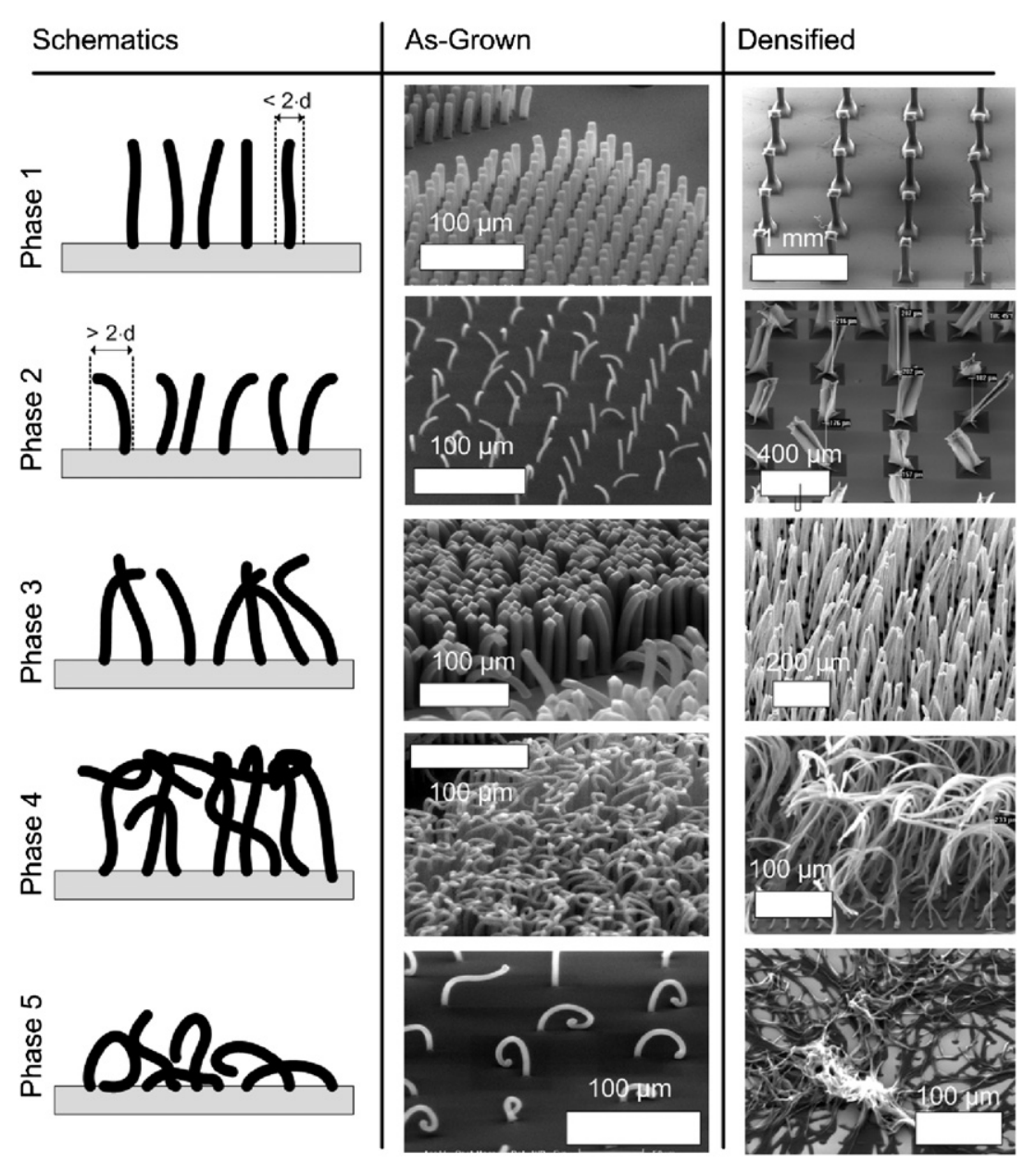

Figure 8. Classification of the hierarchical ordering of arrays of as-grown and densified CNT micropillars, according to the phases described in the text.

On one hand, these data establish guidelines for maintaining a vertical orientation after densification; phase 1 is for instance always retained for pillars with an aspect ratio of 1 . On the other hand, it enables engineering for applications that require entangled micropillar architectures (phases 3-5). Note that in a few cases, pillars that were in phase 2 after growth, straighten out to form phase 1 structures during densification, emphasizing the competition between capillary forces within and between adjacent pillars.

\subsection{Mechanical properties of densified CNT micropillars}

Due to their higher packing density, the densified CNT micropillars have a significantly greater mechanical stiffness than as-grown CNT forests. We quantified this increase by performing axial compression tests of identical arrays of cylindrical micropillars (100 $\mu \mathrm{m}$ diameter as-grown, $200 \mu \mathrm{m}$ height), before and after densification. The pillars were plasma etched before densification, and the DF was approximately 17. The densified pillars are shown in figure 10(a).

The structures were tested in a custom-built microcompression testing machine using a tapered steel tip with diameter of $0.55 \mathrm{~mm}$, fixed in series to a load cell (Futek) that is rigidly mounted to a linear stage. The tip cross-section was chosen to test seven cylindrical CNT microstructures in parallel. The test was performed in position control mode where compression proceeds to $\approx 25 \%$ strain $(50 \mu \mathrm{m})$. At this value of compression strain, the pillars experience both elastic and plastic deformation. The load-displacement and stressstrain curves are shown in figure $10(b)$. Elastic strains of $17 \%$ and $5 \%$ are recovered during unloading of the as-grown and densified structures, respectively.

According to standard methods [36, 37], Young's modulus is calculated using the slope of the unloading curve and the measured total area of the tested structures. Densification increases Young's modulus of the micropillars from 1.56 MPa to $2.24 \mathrm{GPa}$. The latter value is comparable to typical microfabrication polymers including SU-8 $(E=2-4 \mathrm{GPa}$ [38]) and PMMA ( $E=2-5 \mathrm{GPa}$ [39]). Notably, while the reduction in the cross-sectional area is 17 , the modulus increases by a factor of more than 1400 . The great increase in the modulus can be attributed to the change in the collective loading mechanism of individual tubes within the forest, which is caused by the reduction in CNT-CNT spacing [21]. Briefly, due to their waviness, the CNTs within the forests can be modeled as a number of springs in parallel, which support one another at periodic contact points. Thus individual CNTs within a forest are not bearing pure compression 

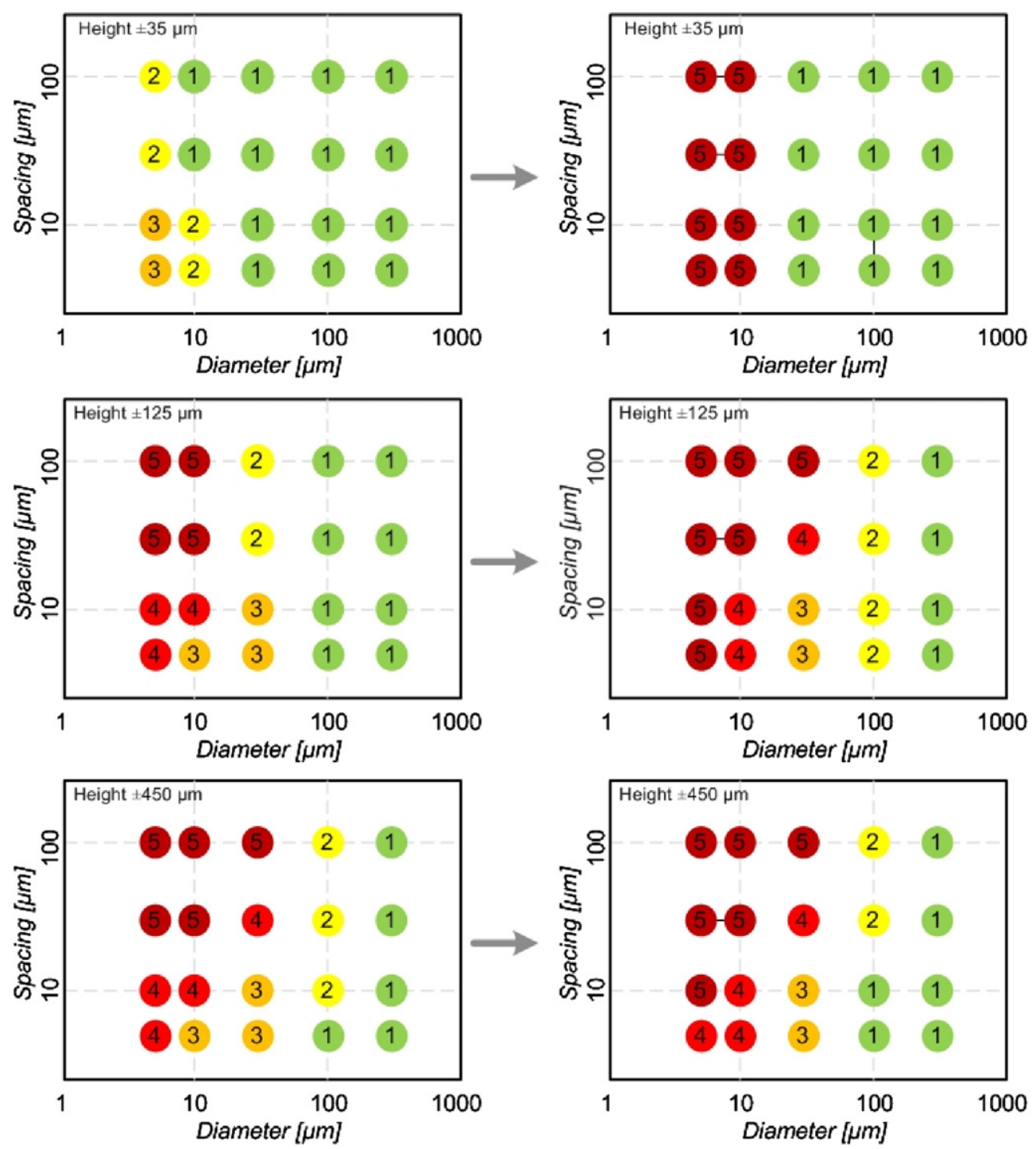

Figure 9. Influence of the diameter, length and spacing on the phase of round micropillar arrays before densification and after glycerol densification. No plasma treatment was applied.

(unlike a rod under compression), and instead they are mostly subject to bending and shearing loads. The CNT-CNT spacing changes the boundary conditions on the individual sections of the springs defining new critical lengths for these spring sections (similar to springs with variable winding diameter and pitch, or cantilevers with variable lengths), thus modifying their collective stiffness. Because the stiffness scales with the cube of the critical length of the individual CNT sections, densification causes a disproportionate increase in the modulus. The ability of CNT micropillars to withstand large compressive strains (here $25 \%$ ), along with the known high temperature stability of CNTs (e.g., exceeding $600{ }^{\circ} \mathrm{C}$ in air [40]) makes them attractive elements for MEMS devices operating in harsh environments.

\subsection{Electrical properties of densified CNT micropillars}

In addition to being mechanically robust, the densified CNT micropillars can be electrically integrated with conventional microfabrication processing. We demonstrate this by growing CNT forests on patterned TiN electrodes, followed by densification and electrical characterization. The TiN electrodes were deposited by sputtering and patterned by photolithography before the catalyst patterning step. The catalyst film consisting of $10 \mathrm{~nm} \mathrm{Al}_{2} \mathrm{O}_{3}$ and $1 \mathrm{~nm}$ of $\mathrm{Fe}$ was subsequently deposited by e-beam evaporation on top of the TiN electrodes through a second lithography step. The CNTs are grown (figure 11(a)) and densified (figure 11(b)) as described previously. The structures are tested by placing one probe on the TiN electrode pad while the second probe contacts the tip of the CNT micropillar.

Figure 11(b) compares two-point probe measurements of an individual cylindrical CNT micropillar $(D=40 \mu \mathrm{m}$, $L=300 \mu \mathrm{m}$ ), before and after densification. The measured resistance of the individual structure shown in figure 11(a) decreased from 373 to $0.9 \Omega$ due to densification, which is a factor exceeding 400. This measured resistance includes a contribution from the contact resistance between the CNTs and the probes and the series resistance of the $\mathrm{Al}_{2} \mathrm{O}_{3}$ layer. 

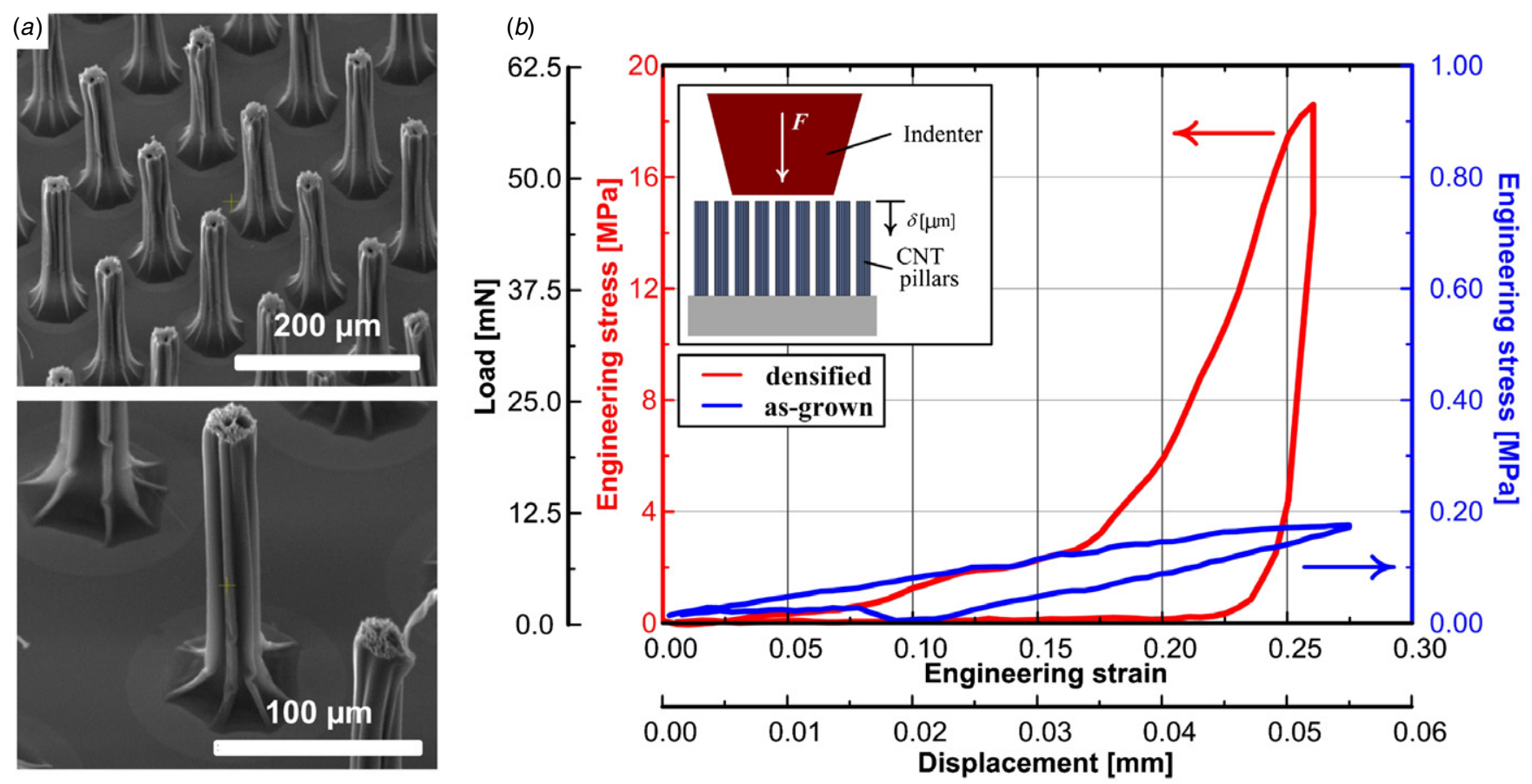

Figure 10. Mechanical properties of as-grown and densified CNT micropillars: (a) SEM images of test structures, which were plasma etched before densification; and $(b)$ stress-strain and load-displacement curves. In $(b)$ the arrows near each curve indicate the corresponding axis scale for stress, while both curves correspond to the same axis scale for applied force. The tip compressed approximately seven pillars simultaneously.
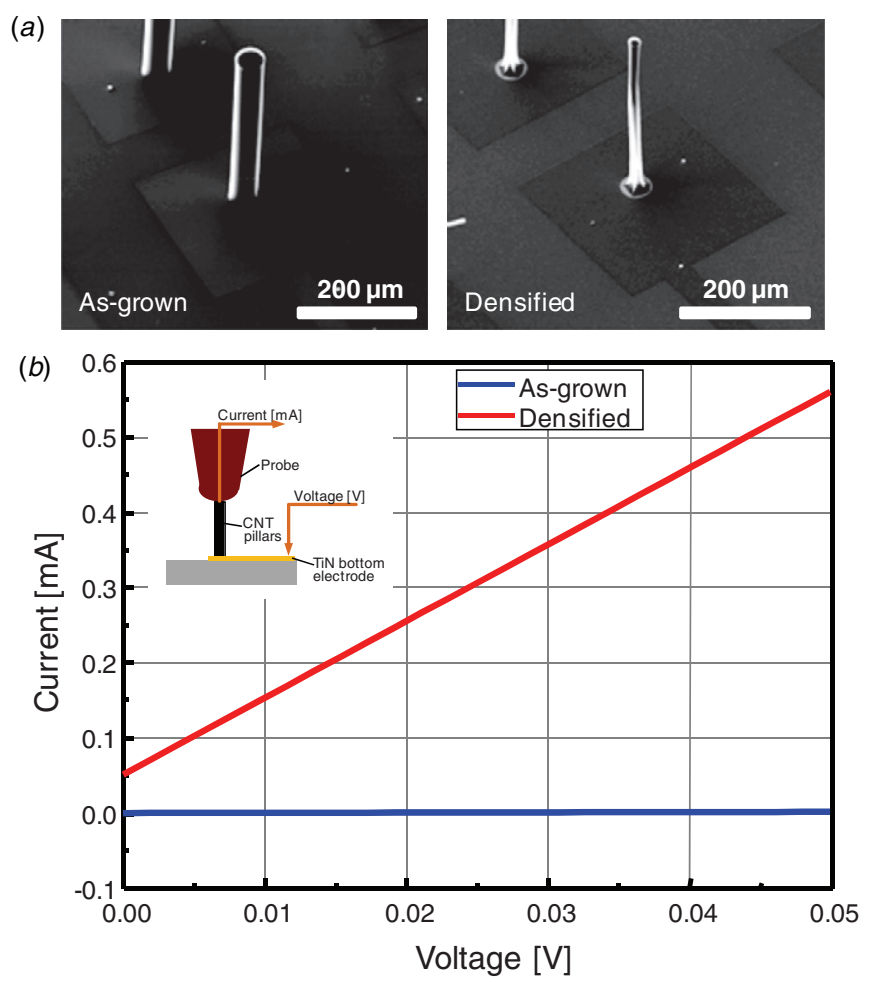

Figure 11. Electrical properties of as-grown and densified CNT micropillars, grown from catalyst deposited on patterned TiN contacts: (a) SEM images; and (b) two-point probe $I-V$ curves.

As discussed for the mechanical stiffness, the enhancement in electrical properties cannot be correlated only with the reduction in cross-sectional area. In fact, if we include the reduction in cross-sectional area due to densification, and calculate the resistivity (not taking into account any change in contact resistance), the decrease in resistivity will be 2300:1. This corresponds to a change from $23 \Omega \mathrm{cm}$ for the as-grown pillar to $9.7 \mathrm{~m} \Omega \mathrm{cm}$ for the densified pillar. The significant reduction in resistance is likely due to: (1) the decrease in contact resistance between the CNTs at the bottom of the structures and the TiN electrode pad, because the CNTs are pulled down onto the TiN electrode as they are densified, thus increasing the effective contact area; (2) improved lateral conduction between CNTs due to densification, which establishes additional electrical paths around critical faults in individual CNTs; and (3) better contact with the top probe which is enabled by the higher stiffness and robustness of the densified pillars. While the as-grown pillars are very fragile and might have been poorly contacted during the measurement, we can still conclude that capillary selfassembly is significantly advantageous for both the electrical and mechanical properties of CNT microstructures.

\section{Conclusions}

We demonstrated that the self-directed elastocapillary densification of CNTs enables the scalable fabrication of robust CNT microstructures from low-density CNT forests grown by CVD. Compared to previous methods of densification by immersion, our new approach allows the densification of closely-spaced structures without undesirable aggregation. Moreover, new insights into this process 
have been gained by combining analytical modeling of elastocapillary densification with the experimental investigation of both the CNT forest geometry and morphology. For instance, the forest aspect ratio, spacing, CNT diameter and plasma treatment all influence the densification of individual pillars, and their collective interactions. These new insights resulted in a set of design guidelines for control of the densification factor, crosssectional shape, pillar straightness and the formation of hierarchical textures. Finally, this process is compatible with microfabrication via direct CNT growth and densification on patterned electrodes. Accordingly, we show an enhancement of three orders of magnitude in both Young's modulus and electrical resistivity of CNT micropillars after densification. Low contact resistance between CNT forests and bottom electrodes is essential for their application in electrical interconnects [41, 42], needle probes [43] and other microsystems. Thus, based on this study, capillary selfassembly could become a key technology for the incorporation of otherwise fragile CNT forests in microdevices.

\section{Acknowledgments}

This research was supported by the College of Engineering and Department of Mechanical Engineering at the University of Michigan, the Belgium Fund for Scientific Research-Flanders (FWO), and the Nanomanufacturing program of the National Science Foundation (CMMI-0927634). We thank Zhaohui Zhong for use of the probe station and Samantha Daly for use of the mechanical compression tester.

\section{References}

[1] Dresselhaus M S, Dresselhaus G and Avouris P 2001 Carbon nanotubes: synthesis, structure, properties, and applications Topics in Applied Physics (New York: Springer)

[2] Harris P J F 2009 Carbon Nanotube Science-Synthesis, Properties, and Applications (Cambridge: Cambridge University Press)

[3] Javey A et al 2003 Ballistic carbon nanotube field-effect transistors Nature $\mathbf{4 2 4} 654-7$

[4] Fischer J E et al 2003 Magnetically aligned single wall carbon nanotube films: preferred orientation and anisotropic transport properties J. Appl. Phys. 93 2157-63

[5] Nihei M et al 2005 Electrical properties of carbon nanotube bundles for future via interconnects Japan. J. Appl. Phys. 44 1626-8

[6] Tong T et al 2008 Height independent compressive modulus of vertically aligned carbon nanotube arrays Nano Lett. 8 511-5

[7] Gwinn J P and Webb R L 2003 Performance and testing of thermal interface materials Microelectron. J. 34 215-22

[8] Fan S S et al 1999 Self-oriented regular arrays of carbon nanotubes and their field emission properties Science $283512-4$

[9] Hart A J and Slocum A H 2006 Rapid growth and flow-mediated nucleation of millimeter-scale aligned carbon nanotube structures from a thin-film catalyst $J$. Phys. Chem. B 110 8250-7

[10] Madou M J 2002 Fundamentals of Microfabrication (Boca Raton, FL: CRC Press)
[11] Hart A J and Slocum A H 2006 Force output, control of film structure, and microscale shape transfer by carbon nanotube growth under mechanical pressure Nano Lett. 6 1254-60

[12] Tawfick S, O'Brien K and Hart A J 2009 Flexible high-conductivity carbon-nanotube interconnects made by rolling and printing Small 5 2467-73

[13] Pint C L et al 2008 Formation of highly dense aligned ribbons and transparent films of single-walled carbon nanotubes directly from carpets ACS Nano 2 1871-8

[14] Wardle B L et al 2008 Fabrication and characterization of ultrahigh-volume-fraction aligned carbon nanotube-polymer composites Adv. Mater. 20 2707-14

[15] Chakrapani N et al 2004 Capillarity-driven assembly of two-dimensional cellular carbon nanotube foams Proc. Natl Acad. Sci. USA 101 4009-12

[16] Liu H et al 2004 Self-assembly of large-scale micropatterns on aligned carbon nanotube films Angew. Chem., Int. Ed. Engl. 43 1146-9

[17] Correa-Duarte M A et al 2004 Fabrication and biocompatibility of carbon nanotube-based 3D networks as scaffolds for cell seeding and growth Nano Lett. 4 2233-6

[18] Futaba D N et al 2006 Shape-engineerable and highly densely packed single-walled carbon nanotubes and their application as super-capacitor electrodes Nat. Mater. 5 987-94

[19] Hayamizu Y et al 2008 Integrated three-dimensional microelectromechanical devices from processable carbon nanotube wafers Nat. Nanotechnol. 3 289-94

[20] Garcia E J et al 2007 Fabrication of composite microstructures by capillarity-driven wetting of aligned carbon nanotubes with polymers Nanotechnology 18165602

[21] Tawfick S et al 2010 Nanocomposite microstructures with tunable mechanical and chemical properties Phys. Chem. Chem. Phys. 12 4446-51

[22] Hutchison D N et al 2010 Carbon nanotubes as a framework for high-aspect-ratio MEMS fabrication J. Microelectromech. Syst. 1975-82

[23] Vaddiraju S et al 2009 Hierarchical multifunctional composites by conformally coating aligned carbon nanotube arrays with conducting polymer ACS Appl. Mater Interfaces $12565-72$

[24] Bico J et al 2004 Elastocapillary coalescence in wet hair Nature $\mathbf{4 3 2} 690$

[25] De Volder M et al 2010 Diverse 3D microarchitectures made by capillary forming of carbon nanotubes $A d v$. Mater. 22 4384-9

[26] Zhao Z, Tawfick S H, Park S J, De Volder M, Hart A J and $\mathrm{Lu} \mathrm{W} 2010$ Bending of nanoscale filament assemblies by elastocapillary densification Phys. Rev. E 82041605

[27] Copic D, Park S-J, Tawfick S, De Volder M and Hart A J 2011 Fabrication of high-aspect-ratio polymer microstructures and hierarchical textures using carbon nanotube composite master molds Lab Chip at press

[28] Py C et al 2007 3D aggregation of wet fibers Europhys. Lett. 7744005

[29] Pokroy B et al 2009 Self-organization of a mesoscale bristle into ordered, hierarchical helical assemblies Science $323237-40$

[30] Namatsu H et al 1995 Dimensional limitations of silicon nanolines resulting from pattern distortion due to surface-tension of rinse water Appl. Phys. Lett. $662655-7$

[31] Journet C et al 2005 Contact angle measurements on superhydrophobic carbon nanotube forests: effect of fluid pressure Europhys. Lett. 71 104-9

[32] Zhao Y P and Fan J G 2006 Clusters of bundled nanorods in nanocarpet effect Appl. Phys. Lett. 88103123

[33] Bedewy M et al 2009 Collective mechanism for the evolution and self-termination of vertically aligned carbon nanotube growth J. Phys. Chem. C 113 20576-82 
[34] Ahn H S et al 2006 Pool boiling experiments on multiwalled carbon nanotube (MWCNT) forests Trans. ASME, J. Heat Transfer 128 1335-42

[35] De Volder M et al 2010 Self-similar organization of arrays of individual carbon nanotubes and carbon nanotube micropillars Microelectron. Eng. 87 1233-8

[36] Shuman D J, Costa A L M and Andrade M S 2007 Calculating the elastic modulus from nanoindentation and microindentation reload curves Mater. Charact. 58 380-9

[37] Oliver W C and Pharr G M 2004 Measurement of hardness and elastic modulus by instrumented indentation: advances in understanding and refinements to methodology J. Mater. Res. 19 3-20

[38] LaFratta C N et al 2007 Multiphoton fabrication Angew. Chem., Int. Ed. Engl. 46 6238-58
[39] Zeng J J et al 2004 Processing and properties of poly(methyl methacrylate)/carbon nano fiber composites Composites B 35 173-8

[40] Meshot E R et al 2009 Engineering vertically aligned carbon nanotube growth by decoupled thermal treatment of precursor and catalyst ACS Nano $32477-86$

[41] Naeemi A et al 2004 Performance comparison between carbon nanotube and copper interconnects for GSI 50th IEEE Int. Electron Devices Meeting (San Francisco, CA) pp 84-6

[42] Liu Z et al 2007 Densification of carbon nanotube bundles for interconnect application 10th Int. Interconnect Technology Conf. pp 201-3

[43] Herwik S et al 2009 Fabrication technology for silicon-based microprobe arrays used in acute and sub-chronic neural recording J. Micromech. Microeng. 19074008 